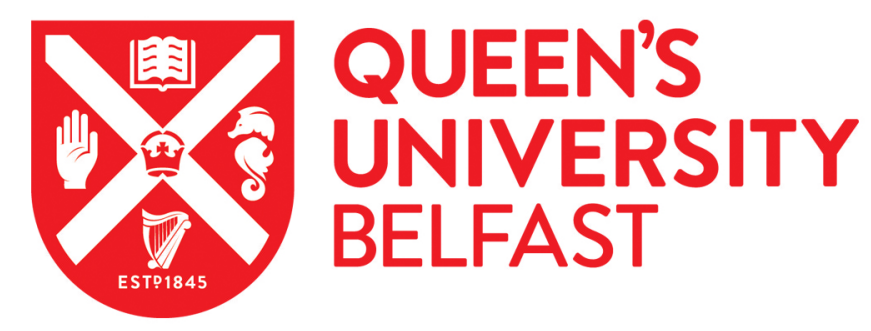

\title{
Finite domination and Novikov homology over strongly Z-graded rings
}

Huettemann, T., \& Steers, L. (2017). Finite domination and Novikov homology over strongly Z-graded rings. Israel Journal of Mathematics, 221(2), 661-685. https://doi.org/10.1007/s11856-017-1569-9

\author{
Published in: \\ Israel Journal of Mathematics
}

Document Version:

Peer reviewed version

Queen's University Belfast - Research Portal:

Link to publication record in Queen's University Belfast Research Portal

\section{Publisher rights}

Copyright 2016 Springer This work is made available online in accordance with the publisher's policies. Please refer to any applicable terms of use of the publisher.

\section{General rights}

Copyright for the publications made accessible via the Queen's University Belfast Research Portal is retained by the author(s) and / or other copyright owners and it is a condition of accessing these publications that users recognise and abide by the legal requirements associated with these rights.

Take down policy

The Research Portal is Queen's institutional repository that provides access to Queen's research output. Every effort has been made to ensure that content in the Research Portal does not infringe any person's rights, or applicable UK laws. If you discover content in the Research Portal that you believe breaches copyright or violates any law, please contact openaccess@qub.ac.uk. 


\title{
FINITE DOMINATION AND NOVIKOV HOMOLOGY OVER STRONGLY $\mathbb{Z}$-GRADED RINGS
}

\author{
THOMAS HÜTTEMANN AND LUKE STEERS
}

\begin{abstract}
Let $L$ be a unital $\mathbb{Z}$-graded ring, and let $C$ be a bounded chain complex of finitely generated $L$-modules. We give a homological characterisation of when $C$ is homotopy equivalent to a bounded complex of finitely generated projective $L_{0}$-modules, generalising known results for twisted LAURENT polynomial rings. The crucial hypothesis is that $L$ is a strongly graded ring.
\end{abstract}

\section{Finite domination over Strongly $\mathbb{Z}$-GRADED RINGS}

Finite domination and Novikov homology. Let $L$ be a unital ring, and let $K$ be a subring of $L$. A bounded chain complex $C$ of (right) $L$-modules is $K$-finitely dominated if $C$, considered as a complex of $K$-modules, is a retract up to homotopy of a bounded complex of finitely generated free $K$-modules; this happens if and only if $C$ is homotopy equivalent, as a $K$-module complex, to a bounded complex of finitely generated projective $K$-modules [Ran85, Proposition 3.2. (ii)]. The following result of RANICKI gives a complete homological characterisation of finite domination in an important special case:

Theorem 1.1 (RANICKI [Ran95, Theorem 2]). Let $R$ be a unital ring, and let $R\left[t, t^{-1}\right]$ denote the LAURENT polynomial ring in the indeterminate $t$. Let $C$ be a bounded chain complex of finitely generated free $R\left[t, t^{-1}\right]$-modules. The complex $C$ is $R$-finitely dominated if and only if both

$$
C \underset{R\left[t, t^{-1}\right]}{\otimes} R\left(\left(t^{-1}\right)\right) \quad \text { and } \quad C \underset{R\left[t, t^{-1}\right]}{\otimes} R((t))
$$

have vanishing homology in all degrees. Here $R((t))=R\left[[t]\left[t^{-1}\right]\right.$ is the ring of formal LAURENT series in $t$, and similarly $R\left(\left(t^{-1}\right)\right)=R\left[\left[t^{-1}\right]\right][t]$ stands for the ring of formal LAURENT series in $t^{-1}$.

The cited paper [Ran95] also contains a discussion of the relevance of finite domination in topology. - The rings $R((t))$ and $R\left(\left(t^{-1}\right)\right)$ are known as Novikov rings. The theorem can be formulated more succinctly: The chain complex $C$ is $R$-finitely dominated if and only if it has trivial NoviKOv homology.

Date: August 26, 2016.

2010 Mathematics Subject Classification. Primary 18G35; Secondary 16W50, $55 \mathrm{U} 15$. 
In the present paper we formulate and prove a surprising strengthening: Theorem 1.1 remains valid if $R\left[t, t^{-1}\right]$ is replaced by an arbitrary strongly $\mathbb{Z}$-graded ring, provided the definition of NovikOV rings is adapted suitably. We start by recalling the requisite definitions.

Definition 1.2. A $\mathbb{Z}$-graded ring is a (unital) ring $L$ equipped with a direct sum decomposition into additive subgroups $L=\bigoplus_{k \in \mathbb{Z}} L_{k}$ such that $L_{k} L_{\ell} \subseteq L_{k+\ell}$ for all $k, \ell \in \mathbb{Z}$, where $L_{k} L_{\ell}$ consists of the finite sums of ring products $x y$ with $x \in L_{k}$ and $y \in L_{\ell}$. The summands $L_{k}$ are called the (homogeneous) components of $L$; elements of $L_{k}$ are called homogeneous of degree $k$. - Following DADE [Dad80] we call $L$ a strongly $\mathbb{Z}$-graded ring, or simply a strongly graded ring, if $L_{k} L_{\ell}=L_{k+\ell}$ for all $k, \ell \in \mathbb{Z}$.

A specific example of a strongly $\mathbb{Z}$-graded ring is $L=R\left[t, t^{-1}\right]$, the ring of LAURENT polynomials; the $n$th component is $\left\{r t^{n} \mid r \in R\right\}$. The reader may wish to keep this motivating example in mind.

We will use the symbol $R_{*}\left[t, t^{-1}\right]=\bigoplus_{k \in \mathbb{Z}} R_{k}$ to denote an arbitrary $\mathbb{Z}$-graded ring in this paper. One may think of the elements of $R_{*}\left[t, t^{-1}\right]$ as formal LAURENT polynomials $\sum_{j=m}^{n} a_{j} t^{j}$ with $a_{j} \in R_{j}$, but note that this is a purely notational device; in general the ring $R_{*}\left[t, t^{-1}\right]$ does not contain an element called $t$. The point of using this notation is that we have a rather suggestive way of denoting various rings and modules constructed from $R_{*}\left[t, t^{-1}\right]$. For example, we can introduce the Novikov rings

$$
R_{*}\left(\left(t^{-1}\right)\right)=\prod_{n \leq 0} R_{n} \oplus \bigoplus_{n>0} R_{n} \quad \text { and } \quad R_{*}((t))=\bigoplus_{n<0} R_{n} \oplus \prod_{n \geq 0} R_{n}
$$

and think of their elements as formal power series $\sum_{j \in \mathbb{Z}} a_{j} t^{j}$ with $a_{j} \in$ $R_{j}$ such that $a_{j}=0$ whenever $j \gg 0$ or $j \ll 0$, respectively.

In any $\mathbb{Z}$-graded ring the unit element is necessarily homogeneous of degree 0 [Dad80, Proposition 1.4] so that $R_{0}$ is a subring of $R_{*}\left[t, t^{-1}\right]$. With these preliminaries in place we can formulate our main result:

Theorem 1.3. Let $R_{*}\left[t, t^{-1}\right]=\bigoplus_{k \in \mathbb{Z}} R_{k}$ be a strongly $\mathbb{Z}$-graded ring, and let $C$ be a bounded chain complex of finitely generated free $R_{*}\left[t, t^{-1}\right]$ modules. The complex $C$ is $R_{0}$-finitely dominated if and only if both

$$
C \underset{R_{*}\left[t, t^{-1}\right]}{\otimes} R_{*}\left(\left(t^{-1}\right)\right) \quad \text { and } \quad C \underset{R_{*}\left[t, t^{-1}\right]}{\otimes} R_{*}((t))
$$

have vanishing homology in all degrees. 
As a special case this says that Theorem 1.1 holds for twisted LAURENT polynomial rings [HK07, Theorem 6], or even for the more general case of crossed products (which are characterised by having homogeneous invertible elements of arbitrary degrees). However, this is not the complete extent of the generalisation as there are strongly graded rings which are not crossed products. Possibly the easiest example to write down is the following: Let $K[A, B, C, D]$ be a polynomial ring over the field $K$, considered as a $\mathbb{Z}$-graded ring by giving $A$ and $C$ degree 1 , and giving $B$ and $D$ degree -1 . Let $R_{*}\left[t, t^{-1}\right]$ be the quotient $K[A, B, C, D] /(A B+C D-1)$; as the relation is homogeneous, this results in a $\mathbb{Z}$-graded ring which is actually strongly graded since $A B+C D=B A+D C=1$ by construction. It can be shown, using ideas from GröBNER basis theory, that the only units are $K^{\times} \subset R_{*}\left[t, t^{-1}\right]$ so that our ring is not a crossed product. Now consider the following 2-step chain complex:

$$
R_{*}\left[t, t^{-1}\right] \stackrel{\left(\begin{array}{l}
1-A \\
1-B
\end{array}\right)}{\longrightarrow} R_{*}\left[t, t^{-1}\right] \oplus R_{*}\left[t, t^{-1}\right] \stackrel{(1-B,-(1-A))}{\longrightarrow} R_{*}\left[t, t^{-1}\right]
$$

The map $1-A$ becomes invertible in $R_{*}((t))$, with inverse $(1-A)^{-1}=$ $\sum_{j \geq 0} A^{j}$; similarly, the map $1-B$ becomes invertible in $R_{*}\left(\left(t^{-1}\right)\right)$. Hence the complex becomes acyclic after tensoring with $R_{*}\left(\left(t^{ \pm 1}\right)\right)$, and Theorem 1.3 asserts that it is in fact $R_{0}$-finitely dominated.

Structure of the paper. For the proof of Theorem 1.3 we combine techniques from strongly graded algebra with homotopy-theoretic methods and homological algebra of bicomplexes. We start by introducing various rings associated to a $\mathbb{Z}$-graded ring, and discuss partitions of unity which are the main technical tool from graded algebra to be used throughout the paper. This will occupy the remainder of $\S 1$.

In $\S 2$ we prove the "if" implication of Theorem 1.3, based on the homotopy-theoretic methods used in [Ran95] for the case of a LAURENT polynomial ring. The organisation follows the pattern laid out by the first author in [Hüt15], where a description of the algebro-geometric background of the procedure is given. It is of interest to note that the $\mathbb{Z}$-graded structure of our ring allows us in Proposition 2.9 to construct complexes of sheaves from the given complex of modules $C$, while the strong grading ensures that certain chain complexes consist of finitely generated projective $R_{0}$-modules, cf. Corollary 2.7 .

In $\S 3$ we attack the reverse implication of Theorem 1.3, using double complex techniques as documented in [Hüt11]. The graded structure is used at various places. Most notably, the definition and the properties of the "algebraic torus", a substitute for the more usual algebraic mapping torus of a self-map of a chain complex, depend crucially on 
extra data which can be chosen only in view of the strong grading. In addition, passage to NovIKOV rings involves a certain "twisting" operation on powers of modules that is defined in terms of the grading.

The results of this paper were obtained as part of the second author's PhD thesis.

Rings associated with $\mathbb{Z}$-graded rings. We make the following conventions for the rest of the paper: All rings, graded or otherwise, are assumed unital and all modules right unless stated otherwise. We let $R_{*}\left[t, t^{-1}\right]$ stand for an arbitrary unital $\mathbb{Z}$-graded ring, with $n$th homogeneous component denoted by $R_{n}$. That is, we have a graded ring $R_{*}\left[t, t^{-1}\right]=\bigoplus_{n \in \mathbb{Z}} R_{n}$. In many cases we will assume this ring to be strongly graded, but will take care to indicate where this hypothesis is really needed.

Given a $\mathbb{Z}$-graded ring $R_{*}\left[t, t^{-1}\right]$, it is known that the unit element 1 must be homogeneous of degree 0 [Dad80, Proposition 1.4]. It is then immediate from the definition that $R_{0}$ is a subring of $R_{*}\left[t, t^{-1}\right]$, and that all the homogeneous components $R_{k}$ are $R_{0}$-bimodules.

Given the $\mathbb{Z}$-graded ring $R_{*}\left[t, t^{-1}\right]$ we define two $\mathbb{Z}$-graded subrings by setting $R_{*}\left[t^{-1}\right]=\bigoplus_{k \leq 0} R_{k}$ and $R_{*}[t]=\bigoplus_{k \geq 0} R_{k}$. These graded rings have trivial components in all positive and negative degrees, respectively. Elements can be thought of as formal polynomials in $t^{-1}$ and $t$, respectively, with the coefficient of $t^{j}$ an element of $R_{j}$.

We can also define the analogues of power series rings, $R_{*}\left[\left[t^{-1}\right]\right]=$ $\prod_{n \leq 0} R_{n}$ and $R_{*}[[t]]=\prod_{n \geq 0} R_{n}$. Elements are of course formal power series in $t^{-1}$ and $t$, respectively, with coefficient of $t^{j}$ an element of $R_{j}$. We have previously defined the Novikov rings $R_{*}\left(\left(t^{-1}\right)\right)$ and $R_{*}((t))$. Note that power series and NoviKOV rings are not considered as graded rings; in fact, they do not admit a natural $\mathbb{Z}$-grading.

The collection of rings fits into the commutative diagram of ring inclusions displayed in Fig. 1 .

\section{Partitions of unity and strongly graded rings.}

Definition 1.4. Given $n \in \mathbb{Z}$, an expression of the form $1=\sum_{j} u_{j} v_{j}$ with $u_{j} \in R_{n}, v_{j} \in R_{-n}$ is called a partition of unity of type $(n,-n)$. This is understood to be a finite sum; we do not specify the summation range unless we need it explicitly.

A partition of unity of type $(n,-n)$ exists if and only if $1 \in R_{n} R_{-n}$. Partitions of unity are our main technical tool; their existence is intimately related to properties of the graded structure of the ring: 


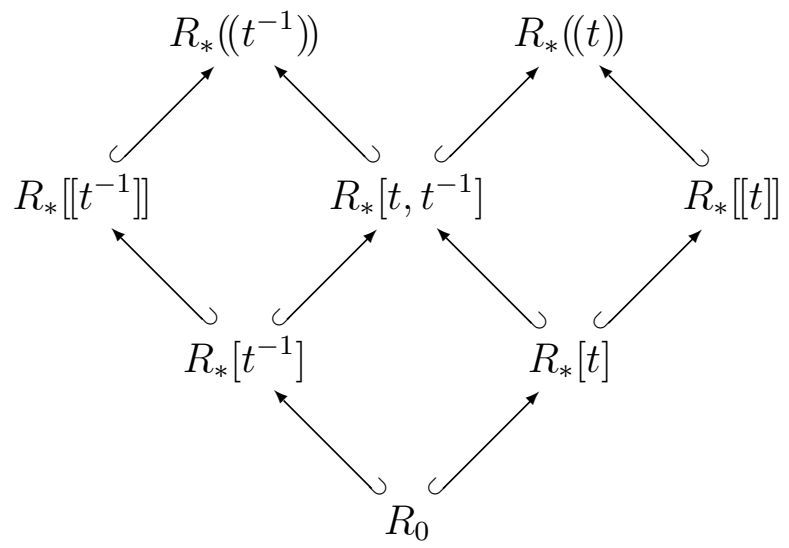

FigurE 1. The collection of rings and their inclusion relation

Proposition 1.5 (Characterisation of strongly graded rings). The following statements are equivalent:

(1) The ring $R_{*}\left[t, t^{-1}\right]$ is strongly graded.

(2) For every $n \in \mathbb{Z}$ there is at least one partition of unity of type $(n,-n)$.

(3) There is at least one partition of unity of type $(1,-1)$, and at least one of type $(-1,1)$.

Proof. For the equivalence of statements (1) and (2) see Proposition 1.6 of [Dad80]. That (2) implies (3) is trivial. For the converse, suppose that $1=\sum_{j=1}^{q} u_{j} v_{j}$ is a partition of unity of type $( \pm 1, \mp 1)$; then for $m \geq 2$ the $q^{m}$ pairs of elements

$$
u_{\mathbf{j}}=u_{j_{1}} u_{j_{2}} \cdots u_{j_{m}} \quad \text { and } \quad v_{\mathbf{j}}=v_{j_{m}} v_{j_{m-1}} \cdots v_{j_{1}},
$$

where $\mathbf{j}=\left(j_{1}, j_{2}, \cdots, j_{m}\right) \in\{1,2, \cdots, q\}^{m}$, form a partition of unity of type $( \pm m, \mp m)$.

The following Proposition is well known; we include a proof because of its fundamental importance for this paper.

Proposition 1.6. If $R_{*}\left[t, t^{-1}\right]$ is strongly graded, then each of the $R_{k}$ is finitely generated projective both as a left $R_{0}$-module and as a right $R_{0}$-module.

Proof. We treat the case of right $R_{0}$-modules only. Let $1=\sum_{j} u_{j} v_{j}$ be a partition of unity of type $(k,-k)$; existence is guaranteed by Proposition 1.5. The maps $g_{j}: R_{k} \rightarrow R_{0}$ with $g_{j}(y)=v_{j} y$ are maps of right $R_{0}$-modules and satisfy $\sum_{j} u_{j} g_{j}(r)=\sum_{j} u_{j} v_{j} r=r$ for any $r \in R_{k}$. Thus $R_{k}$ is a finitely generated projective right $R_{0}$-module by the dual basis lemma, cf. Proposition 12 of [Bou98, §II.2.7]. 
Corollary 1.7. Suppose $R_{*}\left[t, t^{-1}\right]$ is strongly graded. Then any projective left or right $R_{*}\left[t, t^{-1}\right]$-module is also projective when considered as a left or right $R_{0}$-module.

Given numbers $q, p \in \mathbb{Z}$ we define the symbols

$$
t^{q} \cdot R_{*}\left[t^{-1}\right]=\bigoplus_{j \leq q} R_{j} \quad \text { and } \quad t^{-p} \cdot R_{*}[t]=\bigoplus_{j \geq-p} R_{j} ;
$$

the former is an $R_{*}\left[t^{-1}\right]$-bimodule, the latter an $R_{*}[t]$-bimodule. The induced $R_{*}\left[t, t^{-1}\right]$-modules behave as expected in the strongly graded case:

Lemma 1.8. Let $q, p \in \mathbb{Z}$. The $R_{*}\left[t, t^{-1}\right]$-linear maps

$$
\gamma: t^{q} \cdot R_{*}\left[t^{-1}\right] \underset{R_{*}\left[t^{-1}\right]}{\otimes} R_{*}\left[t, t^{-1}\right] \longrightarrow R_{*}\left[t, t^{-1}\right], \quad r \otimes s \mapsto r s
$$

and

$$
\alpha: t^{-p} \cdot R_{*}[t] \underset{R_{*}[t]}{\otimes} R_{*}\left[t, t^{-1}\right] \longrightarrow R_{*}\left[t, t^{-1}\right], \quad r \otimes s \mapsto r s
$$

are isomorphisms provided the ring $R_{*}\left[t, t^{-1}\right]$ is strongly graded.

Proof. Suppose $R_{*}\left[t, t^{-1}\right]$ is strongly graded. Then we may choose a partition of unity of type $(-p, p)$, say $1=\sum_{j} u_{j} v_{j}$ with $u_{j} \in R_{-p}$ and $v_{j} \in R_{p}$. The $R_{*}\left[t, t^{-1}\right]$-linear map

$$
\beta: R_{*}\left[t, t^{-1}\right] \longrightarrow t^{-p} \cdot R_{*}[t] \underset{R_{*}[t]}{\otimes} R_{*}\left[t, t^{-1}\right], \quad r \mapsto \sum_{j} u_{j} \otimes v_{j} r
$$

satisfies $\alpha \beta(r)=\sum_{j} u_{j} v_{j} r=r$ so that $\alpha \beta=\mathrm{id}$. Also

$$
\beta \alpha(r \otimes s)=\beta(r s)=\sum_{j} u_{j} \otimes v_{j} r s \underset{(*)}{=} \sum_{j} u_{j} v_{j} r \otimes s=r \otimes s
$$

(where the equality labelled $(*)$ is true since $v_{j} r \in R_{*}[t]$ for any $r \in$ $\left.t^{-p} \cdot R_{*}[t]\right)$, whence $\beta \alpha=\mathrm{id}$. - The case of $\gamma$ is similar.

The proof of Proposition 1.6 applies with minor modifications to give the following result:

Lemma 1.9. Suppose that $R_{*}\left[t, t^{-1}\right]$ is strongly graded. Then $t^{q} \cdot R_{*}\left[t^{-1}\right]$ is a finitely generated projective left and right $R_{*}\left[t^{-1}\right]$-module. Similarly, $t^{-p} \cdot R_{*}[t]$ is a finitely generated projective left and right $R_{*}[t]-$ module. 


\section{Trivial Novikov homology implies finite Domination}

Sheaves and their cohomology. We will have occasion to study diagrams of the form

$$
\mathfrak{M}=\left(M^{-} \stackrel{\mu^{-}}{\longrightarrow} M \stackrel{\mu^{+}}{\longleftarrow} M^{+}\right)
$$

the entries will be modules, or chain complexes of modules. The maps $\mu^{-}$and $\mu^{+}$are called the structure maps of $\mathfrak{M}$. A map of diagrams consists of a triple of maps $\left(f^{-}, f, f^{+}\right)$which is compatible with the structure maps of source and target.

Definition 2.2. Let as before $R_{*}\left[t, t^{-1}\right]$ be a $\mathbb{Z}$-graded ring. A pre-sheaf is a diagram $\mathfrak{M}$ of the form (2.1) where $M^{-}$is an $R_{*}\left[t^{-1}\right]$-module, $M$ is an $R_{*}\left[t, t^{-1}\right]$-module, $M^{+}$is an $R_{*}[t]$-module, $f^{-}$is $R_{*}\left[t^{-1}\right]$-linear and $f^{+}$is $R_{*}[t]$-linear. The pre-sheaf $\mathfrak{M}$ is called a sheaf if the adjoints of the structure maps $f^{-}$and $f^{+}$are isomorphisms of $R_{*}\left[t, t^{-1}\right]$-modules:

$$
M^{-} \underset{R_{*}\left[t^{-1}\right]}{\otimes} R_{*}\left[t, t^{-1}\right] \stackrel{\cong}{\longrightarrow} M \stackrel{\cong}{\longleftarrow} M^{+} \underset{R_{*}[t]}{\otimes} R_{*}\left[t, t^{-1}\right]
$$

Of particular importance will be the pre-sheaves

$$
\mathcal{O}(q, p)=\left(t^{q} \cdot R_{*}\left[t^{-1}\right] \underset{\iota_{q}}{\stackrel{\subset}{\longrightarrow}} R_{*}\left[t, t^{-1}\right] \underset{p^{\iota}}{\stackrel{\supset}{\supset}} t^{-p} \cdot R_{*}[t]\right)
$$

which depend on the numbers $q, p \in \mathbb{Z}$. In case $R_{*}\left[t, t^{-1}\right]$ is strongly graded these pre-sheaves are actually sheaves by Lemma 1.8, and are then called twisting sheaves.

Back to a general diagram $\mathfrak{M}$ of modules of the form (2.1), we define:

Definition 2.4. The $R_{0}$-module chain complex

$$
H(\mathfrak{M})=\left(M \stackrel{-f^{-}+f^{+}}{\longleftarrow} M^{-} \oplus M^{+}\right)
$$

(concentrated in chain degrees -1 and 0 ) is called the cohomology chain complex of $\mathfrak{M}$. We write $H^{q}(\mathfrak{M})$ for the $(-q)$ th homology of $H(\mathfrak{M})$.

In fact, $H^{q}(\mathfrak{M})=\lim ^{q}(\mathfrak{M})$. - The definitions of pre-sheaf and sheaf apply to chain complexes instead of modules mutatis mutandis; in effect, a (pre-)sheaf of chain complexes is the same as a chain complex of (pre-)sheaves. Given any diagram of chain complexes $\mathfrak{N}=$ $\left(N^{-} \stackrel{g^{-}}{\longrightarrow} N \stackrel{g^{+}}{\longleftarrow} N^{+}\right)$we obtain a double complex $H(\mathfrak{N})$ by applying the cohomology chain complex construction levelwise. (The double complex is concentrated in columns -1 and 0 , and has commuting differentials.) 
Definition 2.5. Given a diagram of chain complexes $\mathfrak{N}$ we define its hypercohomology complex $\mathbb{H}(\mathfrak{N})$ by setting $\mathbb{H}(\mathfrak{N})=$ Tot $H(\mathfrak{N})$, the totalisation of $H(\mathfrak{N})$.

The totalisation is the usual one: $\mathbb{H}(\mathfrak{N})_{n}=N_{n}^{-} \oplus N_{n}^{+} \oplus N_{n+1}$, with differential induced by $-g^{-}, g^{+}$, the differentials of $N^{-}$and $N^{+}$, and the negative of the differential of $N$. Up to shift and sign conventions $\mathbb{H}(\mathfrak{N})$ is the mapping cone of the map $-g^{-}+g^{+}$.

Proposition 2.6. Let $R_{*}\left[t, t^{-1}\right]$ be a $\mathbb{Z}$-graded ring, and let $q, p \in \mathbb{Z}$.

(1) For $p+q \geq 0$, the complex $H(\mathcal{O}(q, p))$ is homotopy equivalent to the chain complex having $\bigoplus_{k=-p}^{q} R_{k}$ in chain level 0 as its only non-trivial chain module.

(2) For $p+q=-1$, the complex $H(\mathcal{O}(q, p))$ is contractible.

(3) For $p+q \leq-2$, the complex $H(\mathcal{O}(q, p))$ is homotopy equivalent to the chain complex having $\bigoplus_{k=q+1}^{-p-1} R_{k}$ in chain level -1 as its only non-trivial chain module.

Proof. We consider the case $p+q \geq 0$ only, the others being similar (and quite irrelevant for our purposes). It is enough to show that the $R_{0}$-module sequence

$0 \longrightarrow \bigoplus_{k=-p}^{q} R_{k} \underset{\rho}{\stackrel{\Delta}{\longrightarrow}} t^{q} \cdot R_{*}\left[t^{-1}\right] \oplus t^{-p} \cdot R_{*}[t] \frac{-\iota_{q}+p \iota}{\stackrel{----}{\hookrightarrow}} R_{*}\left[t, t^{-1}\right] \longrightarrow 0$

is split exact, where $\iota_{q}$ and ${ }_{p} \iota$ denote the inclusions, and where $\Delta$ is the "diagonal" map $r \mapsto(r, r)$; the splitting maps $\rho$ and $\sigma$ will be defined presently. - The sequence can be re-written in more explicit terms:

$$
0 \longrightarrow \bigoplus_{k=-p}^{q} R_{k} \stackrel{\Delta}{\longrightarrow} \bigoplus_{k \leq q} R_{k} \oplus \bigoplus_{k \geq-p} R_{k} \stackrel{-\iota_{q}+p^{\iota}}{\longrightarrow} \bigoplus_{k \in \mathbb{Z}} R_{k} \longrightarrow 0
$$

The composition $\left(-\iota_{q}+{ }_{p} \iota\right) \circ \Delta$ is trivial. We define $\sigma$ by the formula

$$
\sigma: \bigoplus_{k \in \mathbb{Z}} R_{k} \longrightarrow \bigoplus_{k \leq q} R_{k} \oplus \bigoplus_{k \geq-p} R_{k}, \quad \sum_{k \in \mathbb{Z}} r_{k} \mapsto\left(-\sum_{k \leq q} r_{k}, \sum_{k \geq q+1} r_{k}\right)
$$

(note that $p+q \geq 0$ implies $q+1>-p$ ) and $\rho$ by

$$
\rho: \bigoplus_{k \leq q} R_{k} \oplus \bigoplus_{k \geq-p} R_{k} \longrightarrow \bigoplus_{k=-p}^{q} R_{k}, \quad\left(\sum_{k \leq q} r_{k}, \sum_{\ell \geq-p} s_{\ell}\right) \mapsto \sum_{\ell=-p}^{q} s_{\ell} .
$$


They satisfy the identities

$$
\begin{aligned}
\rho \circ \Delta & =\mathrm{id}, \\
\sigma \circ\left(-\iota_{q}+{ }_{p} \iota\right)+\Delta \circ \rho & =\mathrm{id}, \\
\left(-\iota_{q}+{ }_{p} \iota\right) \circ \sigma & =\mathrm{id},
\end{aligned}
$$

as can be verified by direct calculation; thus the sequence is split exact as required.

Corollary 2.7. If $R_{*}\left[t, t^{-1}\right]$ is strongly graded then the cohomology chain complex $H(\mathcal{O}(q, p))$ is $R_{0}$-finitely dominated.

Proof. By Proposition 2.6, $H(\mathcal{O}(q, p))$ is homotopy equivalent to a chain complex with at most one non-zero entry consisting of a finite sum of homogeneous components $R_{k}$ of $R_{*}\left[t, t^{-1}\right]$. Since the $R_{k}$ are all finitely generated projective right $R_{0}$-modules by Proposition 1.6 , $H(\mathcal{O}(q, p))$ is $R_{0}$-finitely dominated.

Building chain complexes of pre-sheaves from chain complexes

of modules. Thanks to the graded structure of our ring $R_{*}\left[t, t^{-1}\right]$ one can extend a given chain complex of $R_{*}\left[t, t^{-1}\right]$-modules to a complex of pre-sheaves. We start with the case of a single module homomorphism.

Lemma 2.8. Let $q, p \in \mathbb{Z}$, and let $f: R_{*}\left[t, t^{-1}\right]^{n} \longrightarrow R_{*}\left[t, t^{-1}\right]^{m}$ be an $R_{*}\left[t, t^{-1}\right]$-linear map. For all sufficiently large numbers $p^{\prime}, q^{\prime} \in \mathbb{Z}$ there exists a map of pre-sheaves

$$
\left(f^{-}, f, f^{+}\right): \bigoplus_{k=1}^{n} \mathcal{O}(q, p) \longrightarrow \bigoplus_{k=1}^{m} \mathcal{O}\left(q^{\prime}, p^{\prime}\right),
$$

depending on $q^{\prime}$ and $p^{\prime}$, which has the given $f$ as its middle component. In other words, the module homomorphism $f$ can be extended to a map of pre-sheaves.

Proof. Consider the following diagram, where $q^{\prime}$ and $p^{\prime}$ are, for the moment, unspecified integers:

$$
\begin{gathered}
\bigoplus_{k=1}^{n} t^{q} \cdot R_{*}\left[t^{-1}\right] \stackrel{\iota_{q}}{\longrightarrow} \bigoplus_{k=1}^{n} R_{*}\left[t, t^{-1}\right] \stackrel{p^{\iota}}{\longleftarrow} \bigoplus_{k=1}^{n} t^{-p} \cdot R_{*}[t] \\
\bigoplus_{k=1}^{m} t^{q^{\prime}} \cdot R_{*}\left[t^{-1}\right] \stackrel{\iota_{q^{\prime}}}{\longrightarrow} \bigoplus_{k=1}^{m} R_{*}\left[t, t^{-1}\right] \stackrel{p^{\prime} \iota}{\longleftarrow} \bigoplus_{k=1}^{m} t^{-p^{\prime}} \cdot R_{*}[t]
\end{gathered}
$$

The map $f$ yields $R_{*}\left[t, t^{-1}\right]$-linear maps ${ }_{k} f_{j}: R_{*}\left[t, t^{-1}\right] \longrightarrow R_{*}\left[t, t^{-1}\right]$ by restriction to the $k$ th summand of the source and the $j$ th summand 
of the target, and the (finite) collection of these maps determines $f$. - For now fix indices $j$ and $k$. The element ${ }_{k} f_{j}(1) \in R_{*}\left[t, t^{-1}\right]$ is a finite sum of non-zero homogeneous elements. Let $-a$ be the minimal occurring degree if ${ }_{k} f_{j}(1) \neq 0$, and an arbitrary integer otherwise. As ${ }_{k} f_{j}(r)={ }_{k} f_{j}(1) \cdot r$, the image of $t^{-p} \cdot R_{*}[t]$ under ${ }_{k} f_{j}$ is contained in $t^{-(p+a)} \cdot R_{*}[t] \subseteq R_{*}\left[t, t^{-1}\right]$, hence is contained in $t^{-p^{\prime}} \cdot R_{*}[t]$ provided $p^{\prime}$ is sufficiently large in the sense that $p^{\prime} \geq a+p$. - Allowing arbitrary indices $j$ and $k$ now, we may choose $p^{\prime}$ sufficiently large for all $j$ and $k$. Then the map $f \circ{ }_{p} \iota$ factors as

$$
\bigoplus_{k=1}^{n} t^{-p} \cdot R_{*}[t] \stackrel{f^{+}}{\longrightarrow} \bigoplus_{k=1}^{m} t^{-p^{\prime}} \cdot R_{*}[t] \stackrel{p^{\prime \iota}}{\longrightarrow} \bigoplus_{k=1}^{m} R_{*}\left[t, t^{-1}\right]
$$

where $f^{+}$is actually the map $f$, suitably restricted in source and target. - The component $f^{-}$is dealt with in a similar manner.

Proposition 2.9 (Extending chain complexes of modules to chain complexes of pre-sheaves). Let $C$ be a bounded above chain complex of finitely generated free $R_{*}\left[t, t^{-1}\right]$-modules together with specified isomorphisms $C_{n} \cong R_{*}\left[t, t^{-1}\right]^{k_{n}}$. Then $C$ is the "middle" component of a chain complex of pre-sheaves. More precisely, there exists a chain complex of pre-sheaves $\mathfrak{D}=\left(D^{-} \longrightarrow D \longleftarrow D^{+}\right)$such that

$$
\mathfrak{D}_{n}=\bigoplus_{k_{n}} \mathcal{O}\left(q_{n}, p_{n}\right)
$$

for certain $q_{n}, p_{n} \in \mathbb{Z}$ with $q_{n}+p_{n} \geq 0$, with $D \cong C$ via the specified isomorphisms.

In case $R_{*}\left[t, t^{-1}\right]$ is strongly graded, $\mathfrak{D}$ is a chain complex of sheaves in the sense of Definition 2.2, with $D^{ \pm}$consisting of finitely generated projective $R_{*}\left[t^{ \pm}\right]$-modules.

Proof. We identify the chain modules $C_{n}$ with direct sums $R_{*}\left[t, t^{-1}\right]^{k_{n}}$ via the given isomorphisms. The boundary maps then take the form of homomorphisms $d_{n}: R_{*}\left[t, t^{-1}\right]^{k_{n}} \longrightarrow R_{*}\left[t, t^{-1}\right]^{k_{n-1}}$.

Let $m$ be the maximal index of a non-zero entry of $C$. Choose $q_{m}=p_{m}=0$.

Now for $\ell=m, m-1, m-2, \cdots$ we use Lemma 2.8 to extend the boundary map $d_{\ell}$ to a map of pre-sheaves

$$
\mathfrak{D}_{\ell}=\bigoplus_{k_{\ell}} \mathcal{O}\left(q_{\ell}, p_{\ell}\right) \stackrel{\left(d_{\ell}^{-}, d_{\ell}, d_{\ell}^{+}\right)}{\longrightarrow} \bigoplus_{k_{\ell-1}} \mathcal{O}\left(q_{\ell-1}, p_{\ell-1}\right)=\mathfrak{D}_{\ell-1}
$$

with $q_{\ell-1}+p_{\ell-1} \geq 0$. 
We have defined a (possibly infinite) sequence of maps of pre-sheaves $\left(d_{\ell}^{-}, d_{\ell}, d_{\ell}^{+}\right)$. These maps are actually boundary maps of a chain complex of pre-sheaves. Indeed, $d_{\ell-1} \circ d_{\ell}=0$ easily implies $d_{\ell-1}^{+} \circ d_{\ell}^{+}=0$ and $d_{\ell-1}^{-} \circ d_{\ell}^{-}=0$ as the structure maps of the diagrams $\mathcal{O}\left(q_{\ell}, p_{\ell}\right)$ are injective.

The last sentence of the Proposition holds as the pre-sheaves $\mathcal{O}(q, p)$ are actually sheaves by Lemma 1.8, consisting of projective modules by Lemma 1.9 , if $R_{*}\left[t, t^{-1}\right]$ is strongly graded.

From trivial Novikov homology to finite domination. With the machinery of sheaves set up we can implement the programme of [Hüt15] to prove that trivial NoviKOv homology implies finite domination. The strong grading proves to be crucial in two places. It is the very fact that twisting sheaves are sheaves (and not just pre-sheaves), combined with finiteness of their cohomology, that makes the proof work.

Notation 2.10. Given a chain complex $\mathfrak{D}=\left(D^{-} \longrightarrow D \longleftarrow D^{+}\right)$ of pre-sheaves let $\mathfrak{D}^{+}$denote the diagram of chain complexes

$$
D^{+} \underset{R_{*}[t]}{\otimes} R_{*}\left[t, t^{-1}\right] \longrightarrow D^{+} \underset{R_{*}[t]}{\otimes} R_{*}((t)) \longleftarrow D^{+} \underset{R_{*}[t]}{\otimes} R_{*}[[t]] ;
$$

similarly, let $\mathfrak{D}^{-}$denote the diagram

$$
D^{-} \underset{R_{*}\left[t^{-1}\right]}{\otimes} R_{*}\left[t, t^{-1}\right] \longrightarrow D^{-} \underset{R_{*}\left[t^{-1}\right]}{\otimes} R_{*}\left(\left(t^{-1}\right)\right) \longleftarrow D^{-} \underset{R_{*}\left[t^{-1}\right]}{\otimes} R_{*}\left[\left[t^{-1}\right]\right] .
$$

In addition, we introduce the variants

$$
\mathfrak{D}^{\prime+}=\left(D^{+} \underset{R_{*}[t]}{\otimes} R_{*}\left[t, t^{-1}\right] \longrightarrow 0 \longleftarrow D^{+} \underset{R_{*}[t]}{\otimes} R_{*}[[t]]\right)
$$

and

$$
\mathfrak{D}^{\prime-}=\left(D^{-} \underset{R_{*}\left[t^{-1}\right]}{\otimes} R_{*}\left[t, t^{-1}\right] \longrightarrow 0 \longleftarrow D^{-} \underset{R_{*}\left[t^{-1}\right]}{\otimes} R_{*}\left[\left[t^{-1}\right]\right]\right),
$$

and write $\zeta^{ \pm}: \mathfrak{D}^{ \pm} \longrightarrow \mathfrak{D}^{ \pm}$for the obvious maps of diagrams:

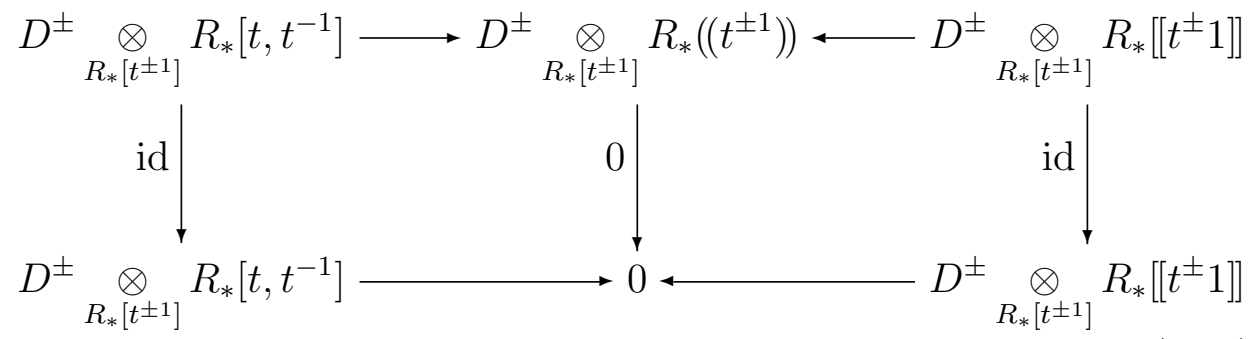


We wish to analyse the hypercohomology complexes of $\mathfrak{D}^{ \pm}$. To begin with, the sequence

$$
0 \longrightarrow R_{*}[t] \stackrel{\Delta}{\longrightarrow} R_{*}\left[t, t^{-1}\right] \oplus R_{*}[[t]] \stackrel{\rho}{\longrightarrow} R_{*}((t)) \longrightarrow 0,
$$

where $\Delta(r)=(r, r)$ and $\rho(r, s)=s-r$, is split exact as a sequence of right $R_{0}$-modules, with splitting maps

$$
R_{*}[t] \stackrel{\kappa}{\longleftarrow} R_{*}\left[t, t^{-1}\right] \oplus R_{*}\left[[t] \stackrel{\lambda}{\longleftarrow} R_{*}((t))\right.
$$

specified by the formulæ

$$
\begin{gathered}
\kappa:\left(\sum_{k \in \mathbb{Z}} r_{k}, \sum_{k \geq 0} s_{k}\right) \mapsto \sum_{k \geq 0} r_{k}, \\
\lambda: \sum_{k \geq n} r_{k} \mapsto\left(-\sum_{k<0} r_{k}, \sum_{k \geq 0} r_{k}\right) .
\end{gathered}
$$

Therefore the sequence (2.12) is exact (but not split) as a sequence of $R_{*}[t]$-bimodules. If the complex $D^{+}$consists of projective $R_{*}[t]$ modules, tensoring $(2.12)$ results in an exact sequence of right $R_{*}[t]$ module chain complexes

$$
\begin{aligned}
0 \longrightarrow D^{+} \underset{R_{*}[t]}{\otimes} R_{*}[t] \longrightarrow D^{+} \underset{R_{*}[t]}{\otimes} R_{*}\left[t, t^{-1}\right] \oplus D^{+} \underset{R_{*}[t]}{\otimes} R_{*}[[t]] \\
\longrightarrow D^{+} \underset{R_{*}[t]}{\otimes} R_{*}((t)) \longrightarrow 0 .
\end{aligned}
$$

This means that $H^{0}\left(\mathfrak{D}^{+}\right)=D^{+} \otimes_{R_{*}[t]} R_{*}[t] \cong D^{+}$and $H^{1}\left(\mathfrak{D}^{+}\right)=$ 0 (levelwise application of $H^{0}$ and $H^{1}$ ). The latter implies that the natural map $\Delta^{+}: H^{0}\left(\mathfrak{D}^{+}\right) \longrightarrow \mathbb{H}\left(\mathfrak{D}^{+}\right)$is a quasi-isomorphism [Hüt15, Lemma 4.2]. - It can be shown by analogous arguments that the natural map $\Delta^{-}: H^{0}\left(\mathfrak{D}^{-}\right) \longrightarrow \mathbb{H}\left(\mathfrak{D}^{-}\right)$is a quasi-isomorphism, with source $D^{-} \otimes_{R_{*}\left[t^{-1}\right]} R_{*}\left[t^{-1}\right] \cong D^{-}$, provided $D^{-}$consists of projective modules. We have shown:

Lemma 2.13. If $D^{+}$consists of projective $R_{*}[t]$-modules the map

$$
\Delta^{+}: H^{0}\left(\mathfrak{D}^{+}\right) \longrightarrow \mathbb{H}\left(\mathfrak{D}^{+}\right)
$$

is a quasi-isomorphism. Similarly, if $D^{-}$consists of projective $R_{*}\left[t^{-1}\right]$ modules the map $\Delta^{-}: H^{0}\left(\mathfrak{D}^{-}\right) \longrightarrow \mathbb{H}\left(\mathfrak{D}^{-}\right)$is a quasi-isomorphism.

Now let us start with a bounded chain complex $C$ of finitely generated free $R_{*}\left[t, t^{-1}\right]$-modules. For each chain module $C_{n} \neq\{0\}$ we choose an isomorphism with $R_{*}\left[t, t^{-1}\right]^{k_{n}}$. Let $\mathfrak{D}=\left(D^{-} \longrightarrow D \longleftarrow D^{+}\right)$denote the resulting complex of pre-sheaves according to Proposition 2.9, and let $\mathfrak{D}^{ \pm}$and $\mathfrak{D}^{ \pm}$be the diagrams defined at the beginning of this 
section. - The structure map $D \longleftarrow D^{+}$has an $R_{*}\left[t, t^{-1}\right]$-linear adjoint, $D \longleftarrow D^{+} \otimes_{R_{*}[t]} R_{*}\left[t, t^{-1}\right]$, which induces a map of diagrams

$$
\mathfrak{D}^{+} \longrightarrow(0 \longrightarrow 0 \longleftarrow D)
$$

upon application of $\mathbb{H}$ this yields a map $\pi^{+}: \mathbb{H}\left(\mathfrak{D}^{+}\right) \longrightarrow D$. We have similarly a map $\pi^{-}: \mathbb{H}\left(\mathfrak{D}^{-}\right) \longrightarrow D$, and analogous maps using $\mathfrak{D}^{\prime \pm}$ denoted $\pi^{\prime \pm}$. All these fit into the commutative diagram displayed in Fig. 2.

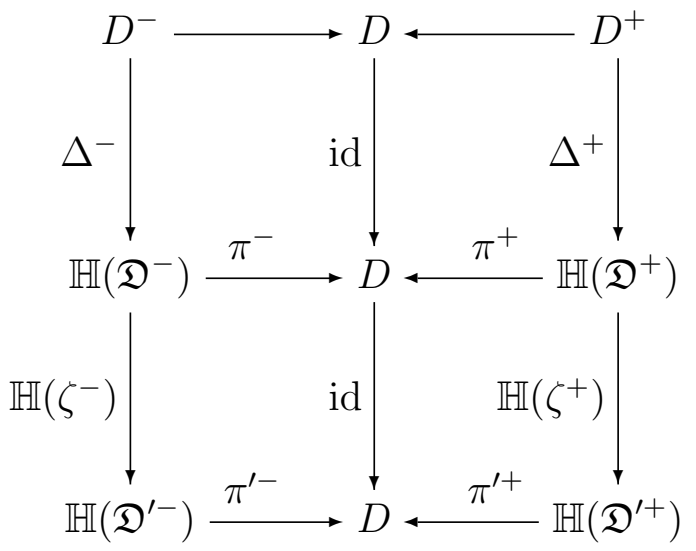

Figure 2. Commutative diagram

Lemma 2.15. If $R_{*}\left[t, t^{-1}\right]$ is strongly graded, and if the two complexes $C \otimes_{R_{*}\left[t, t^{-1}\right]} R_{*}((t))$ and $C \otimes_{R_{*}\left[t, t^{-1}\right]} R_{*}\left(\left(t^{-1}\right)\right)$ have trivial homology, then the maps $\mathbb{H}\left(\zeta^{-}\right)$and $\mathbb{H}\left(\zeta^{+}\right)$are quasi-isomorphisms.

Proof. There is a chain of isomorphisms

$$
\begin{aligned}
& D^{ \pm} \underset{R_{*}\left[t^{ \pm 1}\right]}{\otimes} R_{*}\left(\left(t^{ \pm 1}\right)\right) \cong D^{ \pm} \underset{R_{*}\left[t^{ \pm 1}\right]}{\otimes} R_{*}\left[t, t^{-1}\right] \underset{R_{*}\left[t, t^{-1}\right]}{\otimes} R_{*}\left(\left(t^{ \pm 1}\right)\right) \\
& \cong D \underset{R_{*}\left[t, t^{-1}\right]}{\otimes} R_{*}\left(\left(t^{ \pm 1}\right)\right) \cong C \underset{R_{*}\left[t, t^{-1}\right]}{\otimes} R_{*}\left(\left(t^{ \pm 1}\right)\right),
\end{aligned}
$$

the second one due to the fact that $D$ is a sheaf in the strongly graded setting. By hypothesis the last complex is acyclic. This means that all vertical maps in the diagram (2.11) are quasi-isomorphisms, that is, $\zeta^{ \pm}$consists of quasi-isomorphisms. Hence application of $\mathbb{H}$ results in a quasi-isomorphism $\mathbb{H}\left(\zeta^{ \pm}\right)$by [Hüt15, Lemma 4.2].

Recall that, by construction, $\mathfrak{D}_{n}$ is a finite direct sum of diagrams of the form $\mathcal{O}(q, p)$, with $q+p \geq 0$. It follows from the calculation in Proposition 2.6 that $H^{1}(\mathfrak{D})=0$ (levelwise) so that the inclusion 
$H^{0}(\mathfrak{D}) \longrightarrow \mathbb{H}(\mathfrak{D})$ is a quasi-isomorphism [Hüt15, Lemma 4.2]. With Proposition 1.6 this yields the following result:

Lemma 2.16. The bounded chain complex $H^{0}(\mathfrak{D})$ is quasi-isomorphic to the complex $\mathbb{H}(\mathfrak{D})$. If $R_{*}\left[t, t^{-1}\right]$ is strongly graded, $H^{0}(\mathfrak{D})$ consists of finitely generated projective $R_{0}$-modules.

Proof of Theorem 1.3, "if" part. As before we start with a bounded chain complex $C$ of finitely generated free $R_{*}\left[t, t^{-1}\right]$-modules, and construct a complex of sheaves $\mathfrak{D}=\left(D^{-} \longrightarrow D \longleftarrow D^{+}\right)$according to Proposition 2.9, with $D \cong C$. We will also use the diagrams $\mathfrak{D}^{ \pm}$ and $\mathfrak{D}^{\prime \pm}$ as defined at the beginning of this section.

Our hypothesis now is that $R_{*}\left[t, t^{-1}\right]$ is strongly graded. In this situation all the vertical maps in diagram (2.14) are quasi-isomorphisms, by Lemmas 2.13 and 2.15 . So by applying $\mathbb{H}$ to the rows of the diagram we obtain a chain of maps

$$
H^{0}(\mathfrak{D}) \stackrel{\simeq}{\longrightarrow} \mathbb{H}(\mathfrak{D}) \stackrel{\simeq}{\longrightarrow} \mathbb{H}\left(\mathbb{H}\left(\mathfrak{D}^{\prime-}\right) \stackrel{\pi^{\prime-}}{\longrightarrow} D \stackrel{\pi^{\prime+}}{\longleftarrow} \mathbb{H}\left(\mathfrak{D}^{\prime+}\right)\right)
$$

the first one is a quasi-isomorphism by Lemma 2.16, the second because

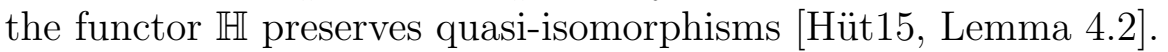

By explicitly spelling out the definitions, we see that the chain complex $\mathbb{H}\left(\mathbb{H}\left(\mathfrak{D}^{\prime-}\right) \stackrel{\pi^{\prime-}}{\longrightarrow} D \stackrel{\pi^{\prime+}}{\longleftarrow} \mathbb{H}\left(\mathfrak{D}^{\prime+}\right)\right)$ contains the complex

$$
\mathbb{H}\left(D^{-} \underset{R_{*}\left[t^{-1}\right]}{\otimes} R_{*}\left[t, t^{-1}\right] \longrightarrow D \longleftarrow D^{+} \underset{R_{*}[t]}{\otimes} R_{*}\left[t, t^{-1}\right]\right)
$$

as a retract. But the diagram $\mathfrak{D}$ is a sheaf, making use of the strong grading again, so the maps $D^{ \pm} \otimes_{R_{*}\left[t^{ \pm 1}\right]} R_{*}\left[t, t^{-1}\right] \longrightarrow D$ are isomorphisms. It follows that the previous chain complex is isomorphic to $\mathbb{H}(D \stackrel{=}{\longrightarrow} D \stackrel{=}{\longrightarrow} D)$, and thus quasi-isomorphic to $D \cong C$.

Combined with (2.17), we thus see that in the derived category of $R_{0}$ the complex $C$ is a retract of $H^{0}(\mathfrak{D})$. Both are bounded complexes of $R_{0}$-projective modules, the former by Corollary 1.7 , the latter by Lemma 2.16. It follows from general theory of derived categories that there are chain maps $\alpha: C \longrightarrow H^{0}(\mathfrak{D})$ and $\beta: H^{0}(\mathfrak{D}) \longrightarrow C$ with $\beta \alpha \simeq$ id. As $H^{0}(\mathfrak{D})$ consists of finitely generated projective $R_{0}$-modules (Lemma 2.16 again), this proves that $C$ is $R_{0}$-finitely dominated as desired.

\section{Finite domination implies trivial Novikov homology}

From now on, and for the remainder of the paper, we suppose that the $\mathbb{Z}$-graded ring $R_{*}\left[t, t^{-1}\right]$ admits a partition of unity $1=\sum_{j} x_{j}^{(-1)} y_{j}^{(1)}$ of type $(-1,1)$, which we choose once and for all. 
Canonical resolution and algebraic tori. For a given $R_{*}\left[t, t^{-1}\right]$ module $C$, or a given chain complex $C$ of such modules, we use the chosen partition of unity to define an $R_{*}\left[t, t^{-1}\right]$-linear map

$$
\begin{aligned}
\mu: C \underset{R_{0}}{\otimes} R_{*}\left[t, t^{-1}\right] \longrightarrow & C \underset{R_{0}}{\otimes} R_{*}\left[t, t^{-1}\right], \\
c \otimes r & \mapsto c \otimes r-\sum_{j} c x_{j}^{(-1)} \otimes y_{j}^{(1)} r .
\end{aligned}
$$

Note that for any $s \in R_{0}$ and any partition of unity $1=\sum_{\ell} u_{\ell} v_{\ell}$ of type $(-1,1)$ there are equalities

$$
\begin{aligned}
& \sum_{j} c x_{j}^{(-1)} \otimes y_{j}^{(1)} s r=\sum_{j, \ell} c x_{j}^{(-1)} \otimes y_{j}^{(1)} s u_{\ell} v_{\ell} r \\
&=\sum_{\ell, j} c x_{j}^{(-1)} y_{j}^{(1)} s u_{\ell} \otimes v_{\ell} r=\sum_{\ell} c s u_{\ell} \otimes v_{\ell} r .
\end{aligned}
$$

Specialising to $u_{\ell}=x_{\ell}^{(-1)}$ and $v_{\ell}=y_{\ell}^{(1)}$ yields that the map $\mu$ is $R_{0}$-balanced, and hence well-defined. On the other hand, specialising to $s=1$ shows that, contrary to appearance, the map $\mu$ does actually not depend on the choice of partition of unity. - It might be worth pointing out that the map $\mu$ cannot be defined in the absence of additional data; the strongly graded structure of the ring enters the picture in a rather subtle form here.

Proposition 3.2 (Canonical resolution). For any $R_{*}\left[t, t^{-1}\right]$-module $M$ there is a sequence of $R_{*}\left[t, t^{-1}\right]$-modules

$$
0 \longrightarrow M \underset{R_{0}}{\otimes} R_{*}\left[t, t^{-1}\right] \stackrel{\mu}{\longrightarrow} M \underset{R_{0}}{\otimes} R_{*}\left[t, t^{-1}\right] \stackrel{\pi}{\longrightarrow} M \longrightarrow 0,
$$

where $\pi(m \otimes r)=m r$ and $\mu$ is as in (3.1). The sequence is natural in $M$. If $R_{*}\left[t, t^{-1}\right]$ is strongly graded then the sequence is split exact as a sequence of right $R_{0}$-modules, and hence is exact (but possibly non-split) as a sequence of right $R_{*}\left[t, t^{-1}\right]$-modules.

Proof. We first note that $\pi \mu=0$ as

$$
\begin{aligned}
\pi \mu(m \otimes r)=\pi(m \otimes r & \left.-\sum_{j} m x_{j}^{(-1)} \otimes y_{j}^{(1)} r\right) \\
& =m r-\sum_{j} m x_{j}^{(-1)} y_{j}^{(1)} r=m r-m 1 r=0 .
\end{aligned}
$$

Let us now suppose that $R_{*}\left[t, t^{-1}\right]$ is strongly graded. In addition to our fixed partition of unity $1=\sum_{\ell_{-1}} x_{\ell_{-1}}^{(-1)} y_{\ell_{-1}}^{(1)}$ we choose for all $k \in \mathbb{Z}$, $k \neq 1$, a partition of unity $1=\sum_{\ell_{-k}} x_{\ell_{-k}}^{(-k)} y_{\ell_{-k}}^{(k)}$ of type $(-k, k)$; as before 
this is understood to be a finite sum with $x_{\ell_{-k}}^{(-k)} \in R_{-k}$ and $y_{\ell_{-k}}^{(k)} \in R_{k}$. Such partitions of unity exist by Proposition $\underline{1.5}$.

We denote by $\iota$ the right $R_{0}$-linear map

$$
\iota: M \longrightarrow M \underset{R_{0}}{\otimes} R_{*}\left[t, t^{-1}\right], \quad m \mapsto m \otimes 1 ;
$$

clearly $\pi \iota=\operatorname{id}_{M}$. Next, we define an $R_{0}$-linear map

$$
\tau: M \underset{R_{0}}{\otimes} R_{*}\left[t, t^{-1}\right] \longrightarrow \underset{R_{0}}{\otimes} R_{*}\left[t, t^{-1}\right]
$$

as $M \otimes_{R_{0}} R_{*}\left[t, t^{-1}\right] \cong \bigoplus_{n \in \mathbb{Z}} M \otimes_{R_{0}} R_{n}$ as a right $R_{0}$-module it will be enough to specify the restrictions $\tau_{n}=\left.\tau\right|_{M \otimes R_{n}}$. For $m \in M$ and $r_{n} \in R_{n}$ these are given by

$$
\tau_{n}\left(m \otimes r_{n}\right)=\left\{\begin{array}{cc}
-\sum_{k=1}^{n} \sum_{\ell_{k}}\left(m x_{\ell_{k}}^{(k)} \otimes y_{\ell_{k}}^{(-k)} r_{n}\right) & \text { if } n>0, \\
0 & \text { if } n=0 \\
\sum_{k=0}^{-n-1} \sum_{j_{-k}}\left(m x_{j_{-k}}^{(-k)} \otimes y_{j_{-k}}^{(k)} r_{n}\right) & \text { if } n<0 .
\end{array}\right.
$$

The map $\tau$ satisfies $\tau \mu=$ id; we will verify $\tau \mu\left(m \otimes r_{n}\right)=m \otimes r_{n}$ for $n \geq 1$, the case $n \leq 0$ being similar. So let $m \in M$ and $r_{n} \in R_{n}$, for some $n \geq 1$. Then

$$
\begin{gathered}
\tau \mu\left(m \otimes r_{n}\right)=\tau\left(m \otimes r_{n}-\sum_{j} m x_{j}^{(-1)} \otimes y_{j}^{(1)} r_{n}\right) \\
=\tau\left(m \otimes r_{n}\right)-\tau\left(\sum_{j} m x_{j}^{(-1)} \otimes y_{j}^{(1)} r_{n}\right) \\
=\tau_{n}\left(m \otimes r_{n}\right)-\tau_{n+1}\left(\sum_{j} m x_{j}^{(-1)} \otimes y_{j}^{(1)} r_{n}\right) .
\end{gathered}
$$

Now by definition

$$
\tau_{n}\left(m \otimes r_{n}\right)=-\sum_{k=1}^{n} \sum_{\ell_{k}}\left(m x_{\ell_{k}}^{(k)} \otimes y_{\ell_{k}}^{(-k)} r_{n}\right)
$$

while

$$
\tau_{n+1}\left(\sum_{j} m x_{j}^{(-1)} \otimes y_{j}^{(1)} r_{n}\right)=-\sum_{k=1}^{n+1}\left(\sum_{\ell_{k}} \sum_{j} m x_{j}^{(-1)} x_{\ell_{k}}^{(k)} \otimes y_{\ell_{k}}^{(-k)} y_{j}^{(1)} r_{n}\right) .
$$


The last term in parentheses, for any fixed $k$, can be simplified:

$$
\begin{aligned}
& \sum_{\ell_{k}} \sum_{j} m x_{j}^{(-1)} x_{\ell_{k}}^{(k)} \otimes y_{\ell_{k}}^{(-k)} y_{j}^{(1)} r_{n}
\end{aligned}
$$

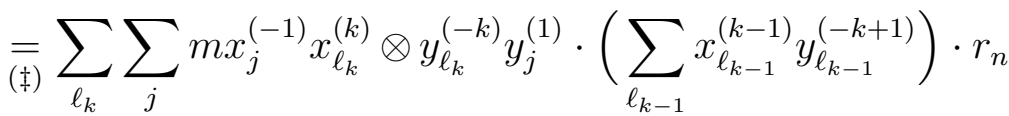

$$
\begin{aligned}
& =\sum_{\ell_{k-1}} \sum_{\ell_{k}} \sum_{j} m x_{j}^{(-1)} x_{\ell_{k}}^{(k)} \otimes y_{\ell_{k}}^{(-k)} y_{j}^{(1)} x_{\ell_{k-1}}^{(k-1)} y_{\ell_{k-1}}^{(-k+1)} r_{n} \\
& \underset{(\dagger)}{=} \sum_{\ell_{k-1}} \sum_{\ell_{k}} \sum_{j} m x_{j}^{(-1)} x_{\ell_{k}}^{(k)} y_{\ell_{k}}^{(-k)} y_{j}^{(1)} x_{\ell_{k-1}}^{(k-1)} \otimes y_{\ell_{k-1}}^{(-k+1)} r_{n} \\
& \underset{(\ddagger)}{=} \sum_{\ell_{k-1}} m x_{\ell_{k-1}}^{(k-1)} \otimes y_{\ell_{k-1}}^{(-k+1)} r_{n}
\end{aligned}
$$

where at $(\dagger)$ we have used that $y_{\ell_{k}}^{(-k)} y_{j}^{(1)} x_{\ell_{k-1}}^{(k-1)} \in R_{0}$, and at $(\ddagger)$ we have used that $\sum_{\ell_{k}} x_{\ell_{k}}^{(k)} y_{\ell_{k}}^{(-k)}=\sum_{\ell_{k-1}} x_{\ell_{k-1}}^{(k-1)} y_{\ell_{k-1}}^{(-k+1)}=\sum_{j} x_{j}^{(-1)} y_{j}^{(1)}=1$. It follows together with the previous expressions that $\tau \mu\left(m \otimes r_{n}\right)$ equals

$$
\begin{array}{r}
-\sum_{k=1}^{n} \sum_{\ell_{k}}\left(m x_{\ell_{k}}^{(k)} \otimes y_{\ell_{k}}^{(-k)} r_{n}\right)+\sum_{k=1}^{n+1} \sum_{\ell_{k-1}}\left(m x_{\ell_{k-1}}^{(k-1)} \otimes y_{\ell_{k-1}}^{(-k+1)} r_{n}\right) \\
=\sum_{\ell_{0}} m x_{\ell_{0}}^{(0)} \otimes y_{\ell_{0}}^{(0)} r_{n}=m \otimes r_{n} .
\end{array}
$$

To show that our sequence (3.3) is split exact when considered as a sequence of $R_{0}$-modules it remains only to prove that $\mu \tau+\iota \pi=$ $\operatorname{id}_{M \otimes R_{*}\left[t, t^{-1}\right]}$. The calculation is similar to the one just finished, making use of existence of partitions of unity in exactly the same manner. We omit the details.

Corollary 3.4. For any chain complex $C$ of $R_{*}\left[t, t^{-1}\right]$-modules there is a quasi-isomorphism cone $(\mu) \stackrel{\sim}{\longrightarrow} C$.

Proof. By the previous Proposition there is a short exact sequence of chain complexes

$$
0 \longrightarrow C \underset{R_{0}}{\otimes} R_{*}\left[t, t^{-1}\right] \stackrel{\mu}{\longrightarrow} C \underset{R_{0}}{\otimes} R_{*}\left[t, t^{-1}\right] \stackrel{\pi}{\longrightarrow} C \longrightarrow 0
$$

Thus the canonical map cone $(\mu) \longrightarrow C$ is a quasi-isomorphism.

Definition 3.5. The mapping cone of $\mu$ in the previous Corollary is called the algebraic torus of $C$ and denoted $\mathfrak{T}(C)$. 
The Mather trick for the algebraic torus. Let $C$ be an $R_{*}\left[t, t^{-1}\right]$ module chain complex, and let $D$ be an $R_{0^{-}}$-module chain complex. Let $\alpha: C \longrightarrow D$ and $\beta: D \longrightarrow C$ be $R_{0}$-linear chain maps and $H$ a chain homotopy such that $H: \beta \alpha \simeq \operatorname{id}_{C}$; that is, $d H+H d=\operatorname{id}_{C}-\beta \alpha$ where $d$ is the differential of $C$. Define

$$
\nu: D \underset{R_{0}}{\otimes} R_{*}\left[t, t^{-1}\right] \longrightarrow D \underset{R_{0}}{\otimes} R_{*}\left[t, t^{-1}\right]
$$

by the formula $\nu=(\alpha \otimes \mathrm{id}) \circ \mu \circ(\beta \otimes \mathrm{id})$. Then the diagram

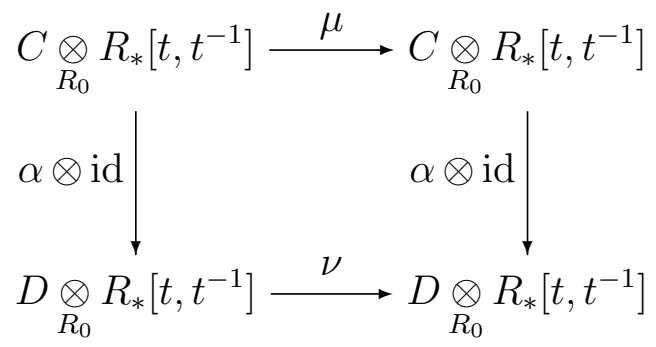

is homotopy commutative with homotopy

$$
J=(\alpha \otimes \mathrm{id}) \circ \mu \circ(H \otimes \mathrm{id}): \nu \circ(\alpha \otimes \mathrm{id}) \simeq(\alpha \otimes \mathrm{id}) \circ \mu .
$$

This homotopy induces a preferred map of $R_{*}\left[t, t^{-1}\right]$-module chain complexes

$$
\alpha_{*}=\left(\begin{array}{cc}
\alpha \otimes \mathrm{id} & 0 \\
J & \alpha \otimes \mathrm{id}
\end{array}\right): \mathfrak{T}(C)=\operatorname{cone}(\mu) \longrightarrow \operatorname{cone}(\nu) .
$$

If $\alpha$ is a quasi-isomorphism and $R_{*}\left[t, t^{-1}\right]$ is strongly graded then $\alpha \otimes$ id is a quasi-isomorphism as well; indeed, the functor $\cdot \otimes_{R_{0}} R_{*}\left[t, t^{-1}\right]$ is exact in the strongly graded case by Proposition 1.6. We obtain the following result analogous to the MATHER trick in the topological context [Ran95, "Whitehead Lemma", §2]:

Lemma 3.7 (MATHER trick). Let $C$ be an $R_{*}\left[t, t^{-1}\right]$-module chain complex, and let $D$ an $R_{0}$-module chain complex. Let $\alpha: C \longrightarrow D$ and $\beta: D \longrightarrow C$ be $R_{0}$-linear chain maps such that $\beta \alpha \simeq \mathrm{id}_{C}$ via a specified homotopy. Then there is a preferred map $\alpha_{*}: \mathfrak{T}(C) \longrightarrow \operatorname{cone}(\nu)$. If in addition $\alpha$ is a quasi-isomorphism and $R_{*}\left[t, t^{-1}\right]$ is strongly graded, $\alpha_{*}: \mathfrak{T}(C) \longrightarrow \operatorname{cone}(\nu)$ is a quasi-isomorphism.

Corollary 3.8. Suppose $R_{*}\left[t, t^{-1}\right]$ is strongly graded. Given a bounded below chain complex $C$ of projective $R_{*}\left[t, t^{-1}\right]$-modules, a bounded below chain complex $D$ of projective $R_{0}$-modules, and an $R_{0}$-homotopy equivalence $\alpha: C \stackrel{\simeq}{\longrightarrow} D$, there is a homotopy equivalence

$$
C \underset{R_{*}\left[t, t^{-1}\right]}{\otimes} R_{*}\left(\left(t^{ \pm 1}\right)\right) \simeq \operatorname{cone}(\nu) \underset{R_{*}\left[t, t^{-1}\right]}{\otimes} R_{*}\left(\left(t^{ \pm 1}\right)\right) .
$$


Proof. From the previous Lemma and Corollary 3.4 we know that there are quasi-isomorphisms $C \longleftarrow \mathfrak{T}(C) \stackrel{\alpha_{*}}{\longrightarrow}$ cone $(\nu)$. As both $C$ and cone $(\nu)$ are bounded below and consist of projective $R_{*}\left[t, t^{-1}\right]$ modules, these two complexes are actually homotopy equivalent. As taking tensor products preserves homotopy equivalences we have proven the claim.

Bicomplexes and truncated powers. We extend our portfolio of homological techniques further by re-writing the complex cone $(\nu)$ as the totalisation of a bicomplex, and by introducing twisted truncated powers.

Let $C$ be an $R_{*}\left[t, t^{-1}\right]$-module chain complex, and let $D$ an $R_{0^{-}}$ module chain complex. Let $\alpha: C \longrightarrow D$ and $\beta: D \longrightarrow C$ be $R_{0^{-}}$ linear chain maps. Define $\nu=(\alpha \otimes$ id $) \circ \mu \circ(\beta \otimes$ id $)$ as in (3.6). Let $\zeta_{n, m}$ denote the $R_{0}$-linear map

$$
D_{m} \underset{R_{0}}{\otimes} R_{n} \longrightarrow D_{m} \underset{R_{0}}{\otimes} R_{n+1}, \quad z \otimes r \mapsto \sum_{j} \alpha\left(\beta(z) x_{j}^{(-1)}\right) \otimes y_{j}^{(1)} r,
$$

and let $E_{\bullet, \bullet}$ denote the bicomplex of right $R_{0}$-modules given by

$$
E_{n, m}=\left(D_{n+m-1} \underset{R_{0}}{\otimes} R_{-n}\right) \oplus\left(D_{n+m} \underset{R_{0}}{\otimes} R_{-n}\right)
$$

with differentials

$$
d_{H}=\left(\begin{array}{cc}
0 & 0 \\
\zeta_{-n, n+m} & 0
\end{array}\right): E_{n, m} \longrightarrow E_{n-1, m}
$$

and

$$
d_{V}=\left(\begin{array}{cc}
-d \otimes \mathrm{id} & 0 \\
\alpha \beta \otimes \mathrm{id} & d \otimes \mathrm{id}
\end{array}\right): E_{n, m} \longrightarrow E_{n, m-1}
$$

where $d$ is the differential of the chain complex $D$.

The totalisation $\operatorname{Tot}\left(E_{\bullet, \bullet}\right)$ is the chain complex with $\operatorname{Tot}\left(E_{\bullet, \bullet}\right)_{\ell}=$ $\bigoplus_{n+m=\ell} E_{n, m}$ and differential $d_{H}+d_{V}$. More explicitly, we have an identification

$$
\begin{aligned}
\operatorname{Tot}\left(E_{\bullet, \bullet}\right)_{\ell}=\bigoplus_{n \in \mathbb{Z}} E_{-n, \ell+n} & =\bigoplus_{n \in \mathbb{Z}}\left(\left(D_{\ell-1} \underset{R_{0}}{\otimes} R_{n}\right)\right) \oplus\left(D_{\ell} \underset{R_{0}}{\otimes} R_{n}\right) \\
& =\left(D_{\ell-1} \underset{R_{0}}{\otimes} R_{*}\left[t, t^{-1}\right]\right) \oplus\left(D_{\ell} \underset{R_{0}}{\otimes} R_{*}\left[t, t^{-1}\right]\right),
\end{aligned}
$$

under which the differential $d=d_{H}+d_{V}$ coincides with the differential of cone $(\nu)$. A straightforward calculation then shows that $d_{H}$ and $d_{V}$ are anti-commuting differentials. We summarise the construction: 
Lemma 3.11. The data listed above yields a bicomplex in the sense that $d_{H} \circ d_{H}=0, d_{H} \circ d_{V}=-d_{V} \circ d_{H}$ and $d_{V} \circ d_{V}=0$. Its totalisation $\operatorname{Tot}\left(E_{\bullet, \bullet}\right)$ is isomorphic to cone $(\nu)$.

We wish to analyse the tensor product cone $(\nu) \otimes_{R_{*}\left[t, t^{-1}\right]} R_{*}((t))$ using the bicomplex above. For this, we need to digress a little and talk about truncated powers, or rather a "twisted" version thereof that takes the graded structure of the ring into account.

Definition 3.12. Given a right $R_{0}$-module $M$, we define the twisted left truncated power of $M$, denoted ${ }^{\mathrm{lt}} \prod_{\sim} M$, by

$$
\prod_{\sim}^{\mathrm{lt}} M=\bigoplus_{n<0}\left(M \underset{R_{0}}{\otimes} R_{n}\right) \oplus \prod_{n \geq 0}\left(M \underset{R_{0}}{\otimes} R_{n}\right)
$$

and the twisted right truncated power of $M$, denoted $\prod_{\sim}^{\mathrm{rt}} M$, by

$$
\prod_{\sim}^{\mathrm{rt}} M=\prod_{n \leq 0}\left(M \underset{R_{0}}{\otimes} R_{n}\right) \oplus \bigoplus_{n>0}\left(M \underset{R_{0}}{\otimes} R_{n}\right) .
$$

We note that ${ }^{\mathrm{lt}} \prod_{\sim} M$ has a right $R_{*}((t))$-module structure; if we write elements of ${ }^{\mathrm{lt}} \prod_{\sim} M$ as formal LAURENT series $\sum_{n \geq m} x t^{n}$ with $x_{n} \in$ $M \otimes_{R_{0}} R_{n}$ and elements of $R_{*}((t))$ as formal LAURENT series $\sum_{n \geq p} r_{n} t^{n}$ with $r_{n} \in R_{n}$, it is given by the obvious multiplication of series formula using $x_{k} r_{n} \in M \otimes_{R_{0}} R_{k+n}$ via the assignment $\left(m \otimes s_{k}\right) \cdot r_{n}=m \otimes\left(s_{k} r_{n}\right)$. - Similarly, $\prod_{\sim}^{\mathrm{rt}} M$ carries a natural right $R_{*}\left(\left(t^{-1}\right)\right)$-module structure.

Proposition 3.13. For a finitely presented $R_{0}$-module $M$, there is an isomorphism of right $R_{*}((t))$-modules

$$
\Phi_{M}: M \underset{R_{0}}{\otimes} R_{*}((t)) \longrightarrow{ }^{\mathrm{lt}} \prod_{\sim} M, \quad m \otimes \sum_{k} r_{k} t^{k} \mapsto \sum_{k}\left(m \otimes r_{k}\right) t^{k}
$$

and an isomorphism of right $R_{*}\left(\left(t^{-1}\right)\right)$-modules

$$
\Psi_{M}: M \underset{R_{0}}{\otimes} R_{*}\left(\left(t^{-1}\right)\right) \longrightarrow \prod_{\sim}^{\mathrm{rt}} M, \quad m \otimes \sum_{k} r_{k} t^{k} \mapsto \sum_{k}\left(m \otimes r_{k}\right) t^{k} .
$$

Both isomorphisms are natural in $M$. 
Proof. We show that $\Phi_{M}$ is bijective, the case of $\Psi_{M}$ being similar. - Suppose first that $M=F$ is free on the basis $e_{1}, e_{2}, \cdots, e_{q}$. Then $F \otimes_{R_{0}} R_{*}((t))$ is a free $R_{*}((t))$-module with $e_{1} \otimes 1, e_{2} \otimes 1, \cdots, e_{q} \otimes 1$ as basis. Thus any $x \in F \otimes_{R_{0}} R_{*}((t))$ can uniquely be written in the form

$$
x=\sum_{j=1}^{q}\left(e_{j} \otimes \sum_{k} r_{j k} t^{k}\right)
$$

with $r_{j k} \in R_{k}$, and $r_{j k}=0$ if $k$ is sufficiently small. Suppose that $x \in \operatorname{ker} \Phi_{F}$ so that

$$
0=\Phi_{F}(x)=\sum_{j=1}^{q} \sum_{k}\left(e_{j} \otimes r_{j k}\right) t^{k}=\sum_{k} \sum_{j=1}^{q}\left(e_{j} \otimes r_{j k}\right) t^{k}
$$

in the twisted left truncated power of $F$. This implies the equality $\sum_{j=1}^{q} e_{j} \otimes r_{j k}=0 \in F \otimes_{R_{0}} R_{k} \subseteq F \otimes_{R_{0}} R_{*}\left[t, t^{-1}\right]$ for all $k$; as the last module is free on basis elements $e_{j} \otimes 1$ we conclude that $r_{j k}=0$ for all $k$ and $j$. Consequently $x=0$ which proves that $\Phi_{F}$ is injective.

Now let $z=\sum_{k \geq n} z_{k} t^{k} \in \prod_{\sim}^{\mathrm{lt}} F$ with $z_{k} \in F \otimes_{R_{0}} R_{k}$; using that $F$ is free on basis elements $e_{j}$ as before we see that we can write $z_{k}$ in the form $z_{k}=\sum_{j} e_{j} \otimes z_{j k}$ with $z_{j k} \in R_{k}$. Then

$$
x=\sum_{j}\left(e_{j} \otimes \sum_{k} z_{j k} t^{k}\right)
$$

is an element of $F \otimes_{R_{0}} R_{*}((t))$ satisfying $\Phi_{F}(x)=z$. Thus $\Phi_{F}$ is seen to be surjective.

For the general case consider a presentation $G \longrightarrow F \longrightarrow M \longrightarrow 0$ of $M$ by finitely generated free modules $F$ and $G$; standard homological algebra, using that the functors $X \mapsto X \otimes_{R_{0}} R_{*}((t))$ and $X \mapsto \prod_{\sim}^{\mathrm{lt}} X$ are right exact, shows that $\Phi_{M}$ is bijective, cf. [Hüt11, Lemma 2.1].

The right truncated totalisation of $E_{\bullet \bullet \bullet}$, denoted $\operatorname{Tot}^{\text {rt }}\left(E_{\bullet}, \bullet\right)$, is the chain complex with

$$
\operatorname{Tot}^{\mathrm{rt}}\left(E_{\bullet, \bullet}\right)_{\ell}=\prod_{n \leq 0} E_{n, \ell-n} \oplus \bigoplus_{n \geq 0} E_{n, \ell-n}
$$


and differential $d_{H}+d_{V}$. Plugging in the definition of $E_{n, m}$ this can be re-written as

$$
\begin{aligned}
& \operatorname{Tot}^{\mathrm{rt}}\left(E_{\bullet}, \bullet\right)_{\ell}=\prod_{n \leq 0}\left(\left(D_{\ell-1} \underset{R_{0}}{\otimes} R_{-n}\right) \oplus\left(D_{\ell} \underset{R_{0}}{\otimes} R_{-n}\right)\right) \\
& \oplus \bigoplus_{n>0}\left(\left(D_{\ell-1} \underset{R_{0}}{\otimes} R_{-n}\right) \oplus\left(D_{\ell} \underset{R_{0}}{\otimes} R_{-n}\right)\right) \\
& =\prod_{\sim}^{\mathrm{lt}} D_{\ell-1} \oplus{ }^{\mathrm{lt}} \prod_{\sim} D_{\ell} \text {; }
\end{aligned}
$$

if the complex $D$ consists of finitely presented $R_{0}$-modules we can thus use Proposition 3.13 to identify $\operatorname{Tot}^{\mathrm{rt}}\left(E_{\bullet}, \bullet\right)_{\ell}$ with the module $\left(D_{\ell-1} \otimes_{R_{0}} R_{*}((t))\right) \oplus\left(\bar{D}_{\ell} \otimes_{R_{0}} R_{*}((t))\right)$. When combined with the isomorphisms cone $(\nu) \otimes_{R_{*}\left[t, t^{-1}\right]} R_{*}((t)) \cong \operatorname{cone}\left(\nu \otimes \operatorname{id}_{\left.R_{*}((t))\right)}\right.$ and

$$
D_{\ell} \underset{R_{0}}{\otimes} R_{*}\left[t, t^{-1}\right] \underset{R_{*}\left[t, t^{-1}\right]}{\otimes} R_{*}((t)) \cong D_{\ell} \underset{R_{0}}{\otimes} R_{*}((t)),
$$

a straightforward calculation with the differentials $d_{H}$ and $d_{V}$ yields:

Proposition 3.14. If $D$ consists of finitely presented $R_{0}$-modules, there is an isomorphism of $R_{*}((t))$-module chain complexes $\operatorname{Tot}^{\mathrm{rt}}\left(E_{\bullet}, \bullet\right) \cong$ $\operatorname{cone}(\nu) \otimes_{R_{*}\left[t, t^{-1}\right]} R_{*}((t))$.

From finite domination to trivial Novikov homology. We are finally in a position to finish the proof of our main result.

Proof of Theorem 1.3, "only if" part. Suppose that the ring $R_{*}\left[t, t^{-1}\right]$ is strongly graded. Let $C$ be a bounded complex of finitely generated free $R_{*}\left[t, t^{-1}\right]$-modules; suppose that $C$ is $R_{0}$-finitely dominated. Then there is a bounded complex $D$ of finitely generated projective $R_{0}$-modules together with a homotopy equivalence $\alpha: C \longrightarrow D$ of $R_{0^{-}}$ module complexes. Let $\beta$ be a homotopy inverse of $\alpha$. According to Corollary 3.8 this data can be used to manufacture a homotopy equivalence $C \otimes_{R_{*}\left[t, t^{-1}\right]} R_{*}((t)) \simeq \operatorname{cone}(\nu) \otimes_{R_{*}\left[t, t^{-1}\right]} R_{*}((t))$, where $\nu$ is a chain complex self-map of $D \otimes_{R_{0}} R_{*}\left[t, t^{-1}\right]$ as in (3.6). We can use Proposition 3.14 to identify cone $(\nu) \otimes_{R_{*}\left[t, t^{-1}\right]} R_{*}((t))$ with $\operatorname{Tot}^{\mathrm{rt}}\left(E_{\bullet \bullet \bullet}\right)$, the right truncated totalisation of the double complex defined in (3.9), as $D$ consists of finitely presented $R_{0}$-modules. The vertical differential of this complex, defined in (3.10), is the mapping cone of $\alpha \beta \otimes \mathrm{id}$. As $\alpha \beta \simeq$ id this means that the columns of $E_{\bullet, \bullet}$ are acyclic, hence $\operatorname{Tot}^{\text {rt }}\left(E_{\bullet \bullet \bullet}\right)$ is acyclic [Hüt11, Proposition 1.2]. This shows that $C \otimes_{R_{*}\left[t, t^{-1}\right]} R_{*}((t))$, being homotopy equivalent to an acyclic complex, has trivial homology. 
To prove that $C \otimes_{R_{*}\left[t, t^{-1}\right]} R_{*}\left(\left(t^{-1}\right)\right)$ has trivial homology too we cannot simply swap the roles of "left" and "right" as we did not analyse whether the rows of $E_{\bullet \bullet}$ are acyclic. Instead, we can quote what we proved so far, applied to the strongly $\mathbb{Z}$-graded ring $\bar{R}_{*}\left[t, t^{-1}\right]$ with $n$th homogeneous component $R_{-n}$ (which as a ring, neglecting the grading, coincides with $\left.R_{*}\left[t, t^{-1}\right]\right)$. We then conclude that $C \otimes_{R_{*}\left[t, t^{-1}\right]} R_{*}\left(\left(t^{-1}\right)\right)=$ $C \otimes_{\bar{R}_{*}\left[t, t^{-1}\right]} \bar{R}_{*}((t))$ has trivial homology as required.

\section{REFERENCES}

[Bou98] Nicolas Bourbaki. Algebra I. Chapters 1-3. Elements of Mathematics (Berlin). Springer-Verlag, Berlin, 1998. Translated from the French, Reprint of the 1989 English translation.

[Dad80] Everett C. Dade. Group-graded rings and modules. Math. Z., 174(3):241-262, 1980.

[HK07] J. A. Hillman and D. H. Kochloukova. Finiteness conditions and $\mathrm{PD}_{r}$-group covers of $\mathrm{PD}_{n}$-complexes. Math. Z., 256(1):45-56, 2007.

[Hüt11] Thomas Hüttemann. Double complexes and vanishing of Novikov cohomology. Serdica Math. J., 37(4):295-304 (2012), 2011.

[Hüt15] Thomas Hüttemann. Vector bundles on the projective line and finite domination of chain complexes. Math. Proc. R. Ir. Acad., 115A(1):Art. 2, $12,2015$.

[Ran85] Andrew Ranicki. The algebraic theory of finiteness obstruction. Math. Scand., 57(1):105-126, 1985.

[Ran95] Andrew Ranicki. Finite domination and Novikov rings. Topology, 34(3):619-632, 1995.

E-mail address: t.huettemann@qub.ac.uk

$U R L$ : www.qub.ac.uk/puremaths/Staff/Thomas Huettemann/

Pure Mathematics Research Centre, School of Mathematics and

Physics, Queen's University Belfast, Belfast BT7 1NN, Northern

IRELAND, UK 